\title{
Use of incretin-based drugs and risk of cholangiocarcinoma: Scandinavian cohort study
}

\author{
Peter Ueda ${ }^{1}$ (D) $\cdot$ Viktor Wintzell $^{1}$ (D) $\cdot$ Mads Melbye $^{2,3,4}$ (D) $\cdot$ Björn Eliasson $^{5}$ (D) $\cdot$ Ann-Marie Svensson $^{5,6}$ (D) $^{-}$ \\ Stefan Franzén ${ }^{6,7}$ (D) - Soffia Gudbjörnsdottir ${ }^{5,6}$ (D) Kristian Hveem $^{8,9}$ (D) Christian Jonasson $^{8,9,10}$ (D) • \\ Henrik Svanström ${ }^{1,2}$ (iD $\cdot$ Björn Pasternak ${ }^{1,2}$ (1)
}

Received: 4 January 2021 / Accepted: 9 April 2021 / Published online: 13 July 2021

(C) The Author(s) 2021, corrected publication 2021

\begin{abstract}
Aims/hypothesis Concerns have been raised regarding a potential association of use of the incretin-based drugs dipeptidyl peptidase 4 (DPP4) inhibitors and glucagon-like peptide-1 (GLP-1)-receptor agonists with risk of cholangiocarcinoma. We examined this association in nationwide data from three countries.

Methods We used data from nationwide registers in Sweden, Denmark and Norway, 2007-2018, to conduct two cohort studies, one for DPP4 inhibitors and one for GLP-1-receptor agonists, to investigate the risk of incident cholangiocarcinoma compared with an active-comparator drug class (sulfonylureas). The cohorts included patients initiating treatment episodes with DPP4 inhibitors vs sulfonylureas, and GLP-1-receptor agonists vs sulfonylureas. We used Cox regression models, adjusted for potential confounders, to estimate hazard ratios from day 366 after treatment initiation to account for cancer latency.

Results The main analyses of DPP4 inhibitors included 1,414,144 person-years of follow-up from 222,577 patients receiving DPP4 inhibitors (median [IQR] follow-up time, 4.5 [2.6-7.0] years) and 123,908 patients receiving sulfonylureas (median [IQR] follow-up time, 5.1 [2.9-7.8] years) during which 350 cholangiocarcinoma events occurred. Use of DPP4 inhibitors, compared with sulfonylureas, was not associated with a statistically significant increase in risk of cholangiocarcinoma (incidence rate 26 vs 23 per 100,000 person-years; adjusted HR, 1.15 [95\% CI 0.90, 1.46]; absolute rate difference 3 [95\% CI -3, 10] events per 100,000 person-years). The main analyses of GLP-1-receptor agonists included 1,036,587 person-years of follow-up from 96,813 patients receiving GLP-1-receptor agonists (median [IQR] follow-up time, 4.4 [2.4-6.9] years) and 142,578 patients receiving sulfonylureas (median [IQR] follow-up time, 5.5 [3.2-8.1] years) during which 249 cholangiocarcinoma events occurred. Use of GLP-1-receptor agonists was not associated with a statistically significant increase in risk of cholangiocarcinoma (incidence rate 26 vs 23 per 100,000 person-years; adjusted HR, 1.25 [95\% CI 0.89, 1.76]; absolute rate difference 3 [95\% CI -5, 13] events per 100,000 patient-years).

Conclusions/interpretation In this analysis using nationwide data from three countries, use of DPP4 inhibitors and GLP-1receptor agonists, compared with sulfonylureas, was not associated with a significantly increased risk of cholangiocarcinoma.
\end{abstract}

Peter Ueda

peter.ueda@ki.se

1 Clinical Epidemiology Division, Department of Medicine, Solna, Karolinska Institutet, Stockholm, Sweden

2 Department of Epidemiology Research, Statens Serum Institut, Copenhagen, Denmark

3 Department of Clinical Medicine, University of Copenhagen, Copenhagen, Denmark

4 Department of Medicine, Stanford University School of Medicine, Stanford, CA, USA

5 Department of Molecular and Clinical Medicine, Institute of Medicine, University of Gothenburg, Gothenburg, Sweden
6 The Swedish National Diabetes Register, Västra Götalandsregionen, Gothenburg, Sweden

7 Health Metrics, Department of Public Health and Community Medicine, Sahlgrenska Academy, University of Gothenburg, Gothenburg, Sweden

8 K.G. Jebsen Center for Genetic Epidemiology, Department of Public Health and Nursing, Faculty of Medicine and Health Science, NTNU—Norwegian University of Science and Technology, Trondheim, Norway

9 HUNT Research Center, Faculty of Medicine, NTNU—Norwegian University of Science and Technology, Levanger, Norway

10 Division of Health Data and Digitalization, The Norwegian Institute of Public Health, Oslo, Norway 


\section{Research in context}

\section{What is already known about this subject?}

- Concerns have been raised regarding a potential increase in the risk of cholangiocarcinoma associated with use of the incretin-based drugs dipeptidyl peptidase 4 (DPP4) inhibitors and glucagon-like peptide-1 (GLP-1)-receptor agonists

What is the key question?

- Is use of DPP4 inhibitors and GLP-1-receptor agonists associated with an increased risk of cholangiocarcinoma?

\section{What are the new findings?}

- In these two cohort studies using Scandinavian nationwide register data, neither use of DPP4 inhibitors nor use of GLP-1-receptor agonists was associated with a significantly increased risk of cholangiocarcinoma, compared with an active comparator (sulfonylureas). The upper limits of the confidence intervals were consistent with absolute rate increases of no more than 10 events per 100,000 person-years for DPP4 inhibitors and no more than 13 events per 100,000 person-years for GLP-1-receptor agonists

How might this impact on clinical practice in the foreseable future?

- Incretin-based drugs were not associated with increased risk of cholangiocarcinoma

Keywords Cholangiocarcinoma · Dipeptidyl peptidase 4 inhibitors · DPP4 inhibitors · Drug safety · GLP-1-receptor-agonists · Glucagon-like peptide-1-receptor agonists · Type 2 diabetes

\section{Abbreviations \\ DPP4 Dipeptidyl peptidase 4 \\ GLP-1 Glucagon-like peptide-1 \\ SGLT2 Sodium-glucose cotransporter 2}

\section{Introduction}

The incretin-based drug classes, dipeptidyl peptidase 4 (DPP4) inhibitors and glucagon-like peptide-1 (GLP-1)receptor agonists, are commonly used for treatment of type 2 diabetes. European and US clinical guidelines now recommend GLP-1-receptor agonists for cardiovascular disease prevention among patients at high risk [1-4].

Recently, concerns have been raised regarding a potential association between incretin-based drugs and cholangiocarcinoma. These concerns were initially based on biological evidence indicating that the incretin system and the GLP-1receptor might play a role in the development of cholangiocarcinoma [5-7], and further highlighted by an observational study using data from the UK Clinical Practice Research Datalink [8] showing that use of DPP4 inhibitors, compared with other second- or third-line glucose-lowering drugs, was associated with an increased risk of cholangiocarcinoma (HR 1.77 [95\% CI 1.04, 3.01]). Analyses of GLP-1-receptor agonists were similarly suggestive of an increased risk although confidence intervals were wide, with only seven events occurring in the exposed group (HR 1.97 [95\% CI $0.83,4.66])$. While the low number of events of cholangiocarcinoma in clinical trials precludes informative analyses [9-14], this safety signal is currently being monitored by the European Medicines Agency [15, 16].

To provide further data on this safety concern, we used nationwide registers in Sweden, Denmark and Norway to assess the association of use of DPP4 inhibitors and GLP-1receptor agonists with risk of cholangiocarcinoma.

\section{Methods}

Data sources Data sources are described in the electronic supplementary material (ESM) Methods. In brief, we used nationwide health and administrative registers in Sweden, Denmark and Norway, including population registers and Statistics Denmark/Statistics Sweden (vital status, demographics, socioeconomic variables), patient registers (comorbidities, outcomes), prescription registers (study drugs, comedications) and the national cancer registers (outcome). The study was approved by the Regional Ethics Committee in Stockholm, Sweden; the Danish Data Protection Agency; and the Regional Committee for Medical and Health Research Ethics (REC Central), Norway. In Denmark, ethical approval is not required for register-based research. Informed consent was not required. 
Study population and exposure definitions We conducted two separate cohort studies for the analyses of DPP4 inhibitors and GLP-1-receptor agonists, respectively. To reduce the risk of confounding by indication, severity of disease and unmeasured participant characteristics, we used an activecomparator design. The active comparator is ideally a drug that is used in the same clinical situation as the study drug while having no association with the outcome. We used sulfonylureas as the active comparator as clinical guidelines used during the study period recommended DPP4 inhibitors and GLP-1-receptor agonists as well as sulfonylureas as secondor third-line glucose-lowering therapies [17], and no concern regarding an increased risk of cholangiocarcinoma with use of sulfonylureas has been raised.

We based the analyses on treatment episodes. In each study cohort, we identified all treatment episodes of new use of the incretin-based drug of interest and of a sulfonylurea during the study period (2007 to the end of 2018 in Sweden and Denmark and 2010 to the end of 2018 in Norway). The date of filling the prescription constituted cohort entry. New use was defined as not having filled a prescription for the same drug at any time prior to cohort entry. The anatomic therapeutic chemical codes for the study drugs are shown in ESM Table 1. Patients who entered the cohort with a prescription for the incretin-based drug were considered as exposed to this drug until the end of follow-up regardless of whether they subsequently switched to or added a sulfonylurea drug. Patients who entered the cohort with a prescription for sulfonylureas and later switched to or added the incretin-based drug were considered as exposed to sulfonylureas from cohort entry to the date of filling the prescription for the incretinbased drug. Thereafter, this subsequent treatment episode with the incretin-based drug of interest was eligible for inclusion, with the date of filling the prescription constituting start of the treatment episode. Hence, a single participant could contribute with an episode of sulfonylurea use followed by an episode of incretin-based drug use, but not vice versa. In patients with two episodes, the episodes never overlapped in time and patients could not have more than one event, hence fulfilling assumptions of statistical independence (the occurrence of one outcome event could not affect the likelihood of another outcome event). We used this exposure categorisation to capture any exposure to the incretin-based drug and as use of sulfonylureas was not expected to be associated with the outcome. In a sensitivity analysis, we constructed cohorts in which each participant could only contribute with one treatment episode; these were defined based on the first treatment episode during the study period.

For each treatment episode, we applied exclusion criteria as defined in ESM Table 2. The exclusion criteria were selected to increase internal validity (rationales are shown in ESM Table 2) and included history of cholangiocarcinoma any time before cohort entry, healthcare visit for any cancer within the previous year, as well as diagnoses of human immunodeficiency virus, hepatitis $\mathrm{B}$ or $\mathrm{C}$, cystic disease of the liver or choledochal duct, primary sclerosing cholangitis, cystic fibrosis, end-stage illness (e.g. cachexia and coma), drug misuse and severe pancreatic disorders (e.g. pancreatic enzyme substitution and major pancreatic surgery). We also excluded those without any specialist care contact or prescription drug use in the previous year (to exclude those with no encounters with the healthcare systems and those who recently immigrated and thus had incomplete covariate data).

Outcome The outcome was incident cholangiocarcinoma, identified from the national cancer registers in each country (ICD 10 codes: C22.1, C23 and C24). These registers collect information on all cancer cases, the majority of which are morphologically verified, and have high completeness and accuracy [18-20].

Statistical analyses We performed separate analyses for the cohorts comparing DPP4 inhibitor vs sulfonylureas use and GLP-1-receptor agonist vs sulfonylureas use. We pooled data from the three countries and used Cox regression models to estimate HRs for the risk of cholangiocarcinoma with use of each of the incretin-based drugs vs sulfonylureas. Patients were followed from the start of the treatment until the outcome event, death, emigration or end of the study period; as described above, treatment episodes with sulfonylureas were also censored at initiation of the incretin-based drug of interest in the respective cohort. The models used days since treatment initiation as the underlying time scale and were adjusted for country; age; sex; place of birth; cohabitation status; history of cardiovascular disease, diabetes complications, cancer (more than 1 year before cohort entry), gallbladder or pancreatic disorders, liver disease, inflammatory bowel disease and alcohol-related disorders; as well as diabetes medications and measures of healthcare utilisation, as measured at cohort entry (ESM Table 3). To account for cancer latency and to mitigate risk of bias due to early symptoms of the outcome that may affect treatment decisions, the HRs of the primary analysis were estimated from 1 year after treatment initiation. This corresponds to the use of a "lag period' commonly employed in studies of cancer outcomes in pharmacoepidemiology [21]. Hence, those diagnosed with cholangiocarcinoma or meeting other censoring criteria during the lag period did not remain in the population that contributed to the estimation of the HR in the primary analysis. We estimated the absolute rate difference assuming a Poisson distribution. We presented the number and proportion of the outcome events by type of cholangiocarcinoma (extrahepatic, ICD 10 codes: C23, C24.0, C24.1; intrahepatic, ICD 10 code: C22.1; uncategorised, ICD 10 codes: C24.8, C24.9). We also performed separate analyses for each country to assess consistency across data sources. In additional analyses, we performed separate analyses by time since treatment initiation ( 1 to $<3$ years; 3 to $<6$ years; $\geq 6$ years). 
Table 1 Participant characteristics at cohort entry for the analyses of DPP4 inhibitors and GLP-1-receptor agonists, respectively

\begin{tabular}{|c|c|c|c|c|}
\hline \multirow[t]{2}{*}{ Characteristic } & \multicolumn{2}{|c|}{ Cohort for analysis of DPP4 inhibitors } & \multicolumn{2}{|c|}{ Cohort for analysis of GLP-1-receptor agonists } \\
\hline & $\begin{array}{l}\text { DPP4 inhibitors } \\
(\mathrm{n}=259,861)\end{array}$ & $\begin{array}{l}\text { Sulfonylureas } \\
(n=141,156)\end{array}$ & $\begin{array}{l}\text { GLP-1-receptor agonists } \\
(\mathrm{n}=120,290)\end{array}$ & $\begin{array}{l}\text { Sulfonylureas } \\
(n=156,744)\end{array}$ \\
\hline \multicolumn{5}{|l|}{ Country } \\
\hline Sweden & $111,812(43.0)$ & $78,368(55.5)$ & $53,071(44.1)$ & $84,196(53.7)$ \\
\hline Denmark & $79,575(30.6)$ & $43,157(30.6)$ & $45,637(37.9)$ & $47,669(30.4)$ \\
\hline Norway & $68,474(26.4)$ & $19,631(13.9)$ & $21,582(17.9)$ & $24,879(15.9)$ \\
\hline Age, mean (SD) & $64.6(11.9)$ & $64.6(12.5)$ & $59.7(10.8)$ & $64.4(12.4)$ \\
\hline Female & $105,369(40.5)$ & $60,808(43.1)$ & $52,315(43.5)$ & $67,057(42.8)$ \\
\hline \multicolumn{5}{|l|}{ Place of birth } \\
\hline Scandinavia & $217,825(83.8)$ & $116,738(82.7)$ & $104,609(87.0)$ & $129,135(82.4)$ \\
\hline Rest of Europe & $16,840(6.5)$ & $9663(6.8)$ & $6739(5.6)$ & $10,930(7.0)$ \\
\hline Outside Europe & $24,851(9.6)$ & $14,520(10.3)$ & $8820(7.3)$ & $16,411(10.5)$ \\
\hline Missing & $345(0.1)$ & $235(0.2)$ & $122(0.1)$ & $268(0.2)$ \\
\hline \multicolumn{5}{|l|}{ Civil status } \\
\hline Married/living with partner & $147,639(56.8)$ & $77,328(54.8)$ & $68,727(57.1)$ & $86,172(55.0)$ \\
\hline Single & $110,813(42.6)$ & $62,244(44.1)$ & $51,138(42.5)$ & $68,854(43.9)$ \\
\hline Missing & $1409(0.5)$ & $1584(1.1)$ & $426(0.4)$ & $1718(1.1)$ \\
\hline \multicolumn{5}{|l|}{ Calendar year } \\
\hline $2007-2009$ & $21,551(8.3)$ & $42,705(30.3)$ & $4625(3.8)$ & $43,443(27.7)$ \\
\hline $2010-2012$ & $64,544(24.8)$ & $46,393(32.9)$ & $32,317(26.9)$ & $50,183(32.0)$ \\
\hline 2013-2015 & $75,697(29.1)$ & $32,092(22.7)$ & $29,927(24.9)$ & $37,443(23.9)$ \\
\hline 2016-2018 & $98,069(37.7)$ & $19,966(14.1)$ & $53,421(44.4)$ & $25,675(16.4)$ \\
\hline \multicolumn{5}{|l|}{ Comorbidities } \\
\hline Cardiovascular disease & $84,648(32.6)$ & $43,224(30.6)$ & $37,538(31.2)$ & $47,529(30.3)$ \\
\hline Diabetic complications & $91,907(35.4)$ & $37,985(26.9)$ & $52,178(43.4)$ & $42,610(27.2)$ \\
\hline Cancer (excl. non-melanoma skin cancer) ${ }^{\mathrm{a}}$ & $13,092(5.0)$ & $6745(4.8)$ & $5100(4.2)$ & $7449(4.8)$ \\
\hline Gall bladder or pancreatic disorders & $11,188(4.3)$ & $6735(4.8)$ & $5902(4.9)$ & $7380(4.7)$ \\
\hline Liver disease & $3645(1.4)$ & $1880(1.3)$ & $2103(1.7)$ & $2084(1.3)$ \\
\hline Inflammatory bowel disease & $5722(2.2)$ & $3030(2.1)$ & $2989(2.5)$ & $3352(2.1)$ \\
\hline Other alcohol-related disorders & $3997(1.5)$ & $2436(1.7)$ & $1979(1.6)$ & $2621(1.7)$ \\
\hline \multicolumn{5}{|l|}{ Healthcare utilisation in last year } \\
\hline Hospitalisation due to type 2 diabetes & $5021(1.9)$ & $3361(2.4)$ & $2924(2.4)$ & $3598(2.3)$ \\
\hline Hospitalisation due to other causes & $51,344(19.8)$ & $28,468(20.2)$ & $21,973(18.3)$ & $30,985(19.8)$ \\
\hline Outpatient contact due to type 2 diabetes & $41,822(16.1)$ & $11,932(8.5)$ & $33,269(27.7)$ & $14,844(9.5)$ \\
\hline Outpatient contact due to other causes & $139,964(53.9)$ & $71,815(50.9)$ & $71,868(59.7)$ & $79,705(50.9)$ \\
\hline \multicolumn{5}{|l|}{ Diabetes drugs in last 6 months } \\
\hline None & $26,663(10.3)$ & $40,538(28.7)$ & $9284(7.7)$ & $41,371(26.4)$ \\
\hline Metformin & $205,113(78.9)$ & $97,129(68.8)$ & $90,921(75.6)$ & $109,892(70.1)$ \\
\hline Insulin & $35,831(13.8)$ & $6075(4.3)$ & $46,347(38.5)$ & $6385(4.1)$ \\
\hline Thiazolidinediones & $9185(3.5)$ & $2332(1.7)$ & $2351(2.0)$ & $2592(1.7)$ \\
\hline Other antidiabetics (glinides, acarbose) & $6647(2.6)$ & $2400(1.7)$ & $2969(2.5)$ & $2707(1.7)$ \\
\hline GLP-1-receptor agonists & $4623(1.8)$ & $1595(1.1)$ & - & - \\
\hline DPP4 inhibitors & - & - & $12,158(10.1)$ & $5541(3.5)$ \\
\hline
\end{tabular}

Numbers are shown as $n(\%)$ unless indicated otherwise

${ }^{a}$ Recorded more than 1 year prior to start of treatment episode 
We performed sensitivity analyses. First, we estimated HRs without a lag period and with a 2 year lag period, respectively. Second, we performed analyses censoring follow-up at initiation of the other incretin-based drug (initiation of GLP-1receptor agonists in analyses of DPP4 inhibitors and vice versa). Third, we additionally adjusted the analyses for calendar year at the start of the treatment episode as a categorical variable (2007-2009; 2010-2012; 2013-2015; 2016 2018). Fourth, we defined the study cohorts based on the first treatment episode during the study period, so that each participant could contribute to the cohort with no more than one treatment episode; here, cohorts were constructed excluding a
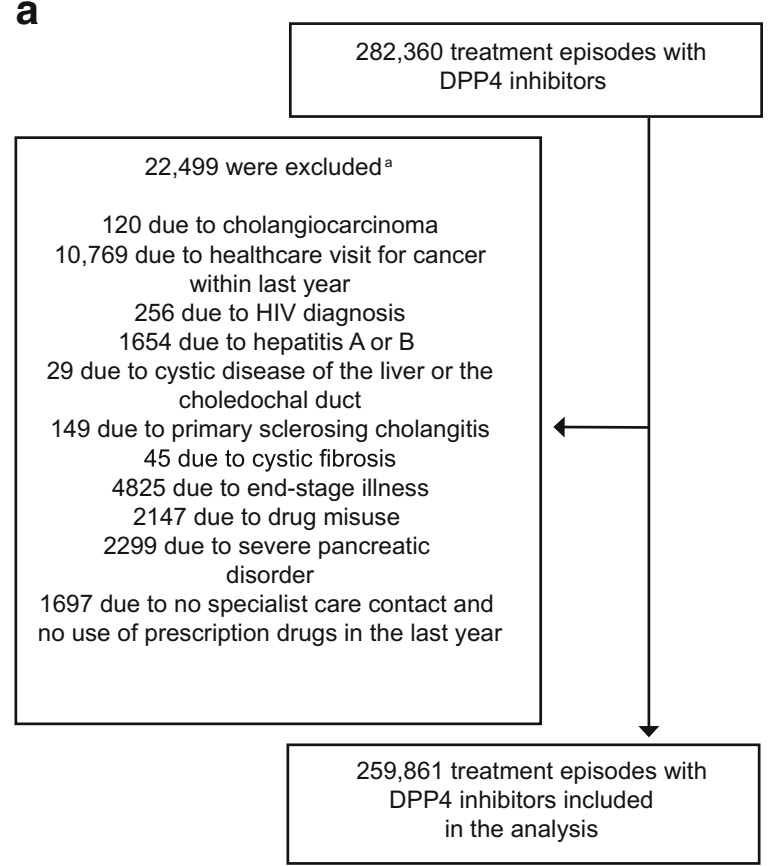

b

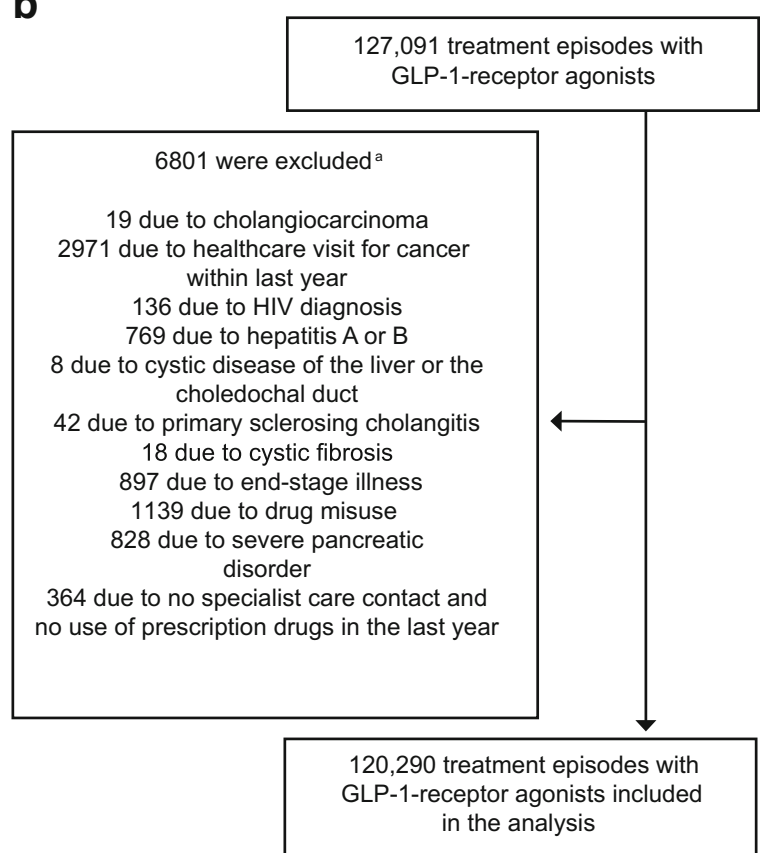

177,123 treatment episodes with sulfonylureas

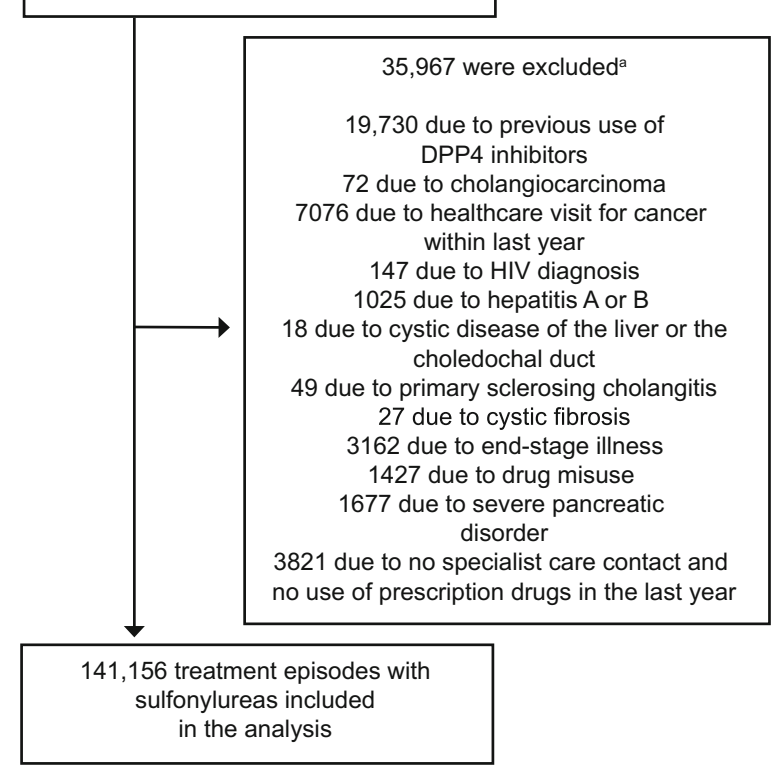

178,080 treatment episodes with sulfonylureas

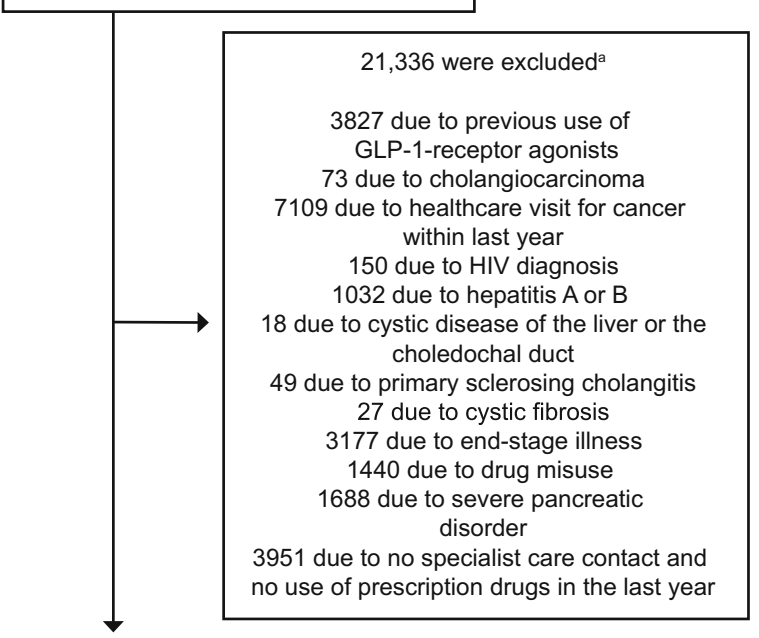

156,744 treatment episodes with sulfonylureas included in the analysis

Fig. 1 Flow chart of treatment episode inclusion in the study cohorts for analyses of (a) DPP4 inhibitors and (b) GLP-1-receptor agonists, respectively. a Participants could be excluded for more than one reason 
patients with previous use of any of the study drugs (the incretin-based drug or sulfonylureas) at cohort entry, with this exclusion being applied in addition to the exclusion criteria described in ESM Table 2. In this analysis patients were considered as exposed to the drug with which they entered the cohort until the end of follow-up. In essence, this analysis was a traditional active-comparator new-user design [22]. Fifth, we performed the analyses using sodium-glucose cotransporter 2 (SGLT2) inhibitors as the comparator: patients with no previous use of either of the study drugs and who initiated the incretin-based drug or an SGLT2 inhibitor from 1 April 2013 and onwards were included, with the date of filling the first prescription constituting cohort entry. Exclusion criteria (ESM Table 2) were applied. Patients were considered as exposed to the drug with which they entered the cohort until the end of follow-up. In addition to the variables shown in ESM Table 3, these analyses were adjusted for history of sulfonylureas use at cohort entry. Finally, we performed the analyses without applying the exclusion criteria related to risk factors of the outcome (ESM Table 2). All statistical analyses were done with SAS version 9.4 (SAS Institute, Cary, NC, USA).

\section{Results}

Study populations For the analyses of DPP4 inhibitors, 401,017 treatment episodes ( $n=259,861$ DPP4 inhibitor episodes; 141,156 sulfonylureas episodes) from 358,341 patients fulfilled the study eligibility criteria (Fig. 1; participant characteristics in Table 1). Among the episodes of DPP4 inhibitor use, mean (SD) age was 64.6 (11.9) and $40.5 \%$ were female. The corresponding numbers for episodes of sulfonylureas use were 64.6 (12.5) years and $43.1 \%$ female. Compared with treatment episodes with sulfonylureas, a larger proportion of the treatment episodes with DPP4 inhibitors were from patients with diabetes complications and with concurrent use of other glucose-lowering medications.

For the analyses of GLP-1-receptor agonists, 277,034 treatment episodes ( $n=120,290$ GLP-1-receptor agonist episodes; 156,744 sulfonylureas episodes) from 252,861 patients were included (Fig. 1; participant characteristics in Table 1). Among episodes of GLP-1-receptor agonist use, mean (SD) age was 59.7 (10.8) years and $43.5 \%$ were female. The corresponding numbers for episodes of sulfonylureas use were 64.4 (12.4) years and $42.8 \%$ female. Also in this study population, a larger proportion of treatment episodes with GLP-1-receptor agonists were from patients with diabetes complications and with concurrent use of other glucose-lowering medications.

Primary analyses Table 2 shows the results of the primary analyses for DPP4 inhibitors and GLP-1-receptor agonists and Fig. 2 shows the cumulative incidence of cholangiocarcinoma. In the analyses of DPP4 inhibitors, 222,577 treatment episodes of DPP4 inhibitor use (median [IQR] follow-up time, 4.5 [2.6-7.0] years) and 123,908 treatment episodes of sulfonylurea use (median [IQR] follow-up time, 5.1 [2.9-7.8] years) remained at risk at 1 year after start of follow-up. During 1,414,144 person-years of follow-up, cholangiocarcinoma occurred among 222 patients with use of DPP4 inhibitors (incidence rate, 26 per 100,000 patient-years) and among 128 patients with use of sulfonylureas (incidence rate, 23 per 100,000 patient-years). The adjusted HR for use of DPP4 inhibitors vs sulfonylureas was 1.15 (95\% CI 0.90 , 1.46) and the absolute difference was $3(95 \%$ CI $-3,10)$ events per 100,000 person-years.

In the analyses of GLP-1-receptor agonists, 96,813 treatment episodes of GLP-1-receptor agonist use (median [IQR] follow-up time, 4.4 [2.4-6.9] years) and 142,578 treatment episodes of sulfonylurea use (median [IQR] follow-up time,

Table 2 Association between use of DPP4 inhibitors and GLP-1-receptor agonists, respectively, and risk of cholangiocarcinoma in the primary analyses

\begin{tabular}{|c|c|c|c|c|c|c|}
\hline Analysis & $\mathrm{n}$ & Events & $\begin{array}{l}\text { Events per } 100,000 \\
\text { person-years }\end{array}$ & $\begin{array}{l}\text { Unadjusted HR } \\
(95 \% \mathrm{CI})\end{array}$ & $\begin{array}{l}\text { Adjusted }^{\mathrm{a}} \mathrm{HR} \\
(95 \% \mathrm{CI})\end{array}$ & $\begin{array}{l}\text { Adjusted }^{\mathrm{a}} \text { absolute rate } \\
\text { difference, events per } 100,000 \\
\text { person-years }\end{array}$ \\
\hline \multicolumn{7}{|c|}{ Analysis of DPP4 inhibitors } \\
\hline DPP4 inhibitors & 222,577 & 222 & 26 & $1.13(0.91,1.41)$ & $1.15(0.90,1.46)$ & $3(-3,10)$ \\
\hline Sulfonylureas & 123,908 & 128 & 23 & [reference] & [reference] & [reference] \\
\hline \multicolumn{7}{|c|}{ Analysis of GLP-1-receptor agonists } \\
\hline $\begin{array}{l}\text { GLP-1-receptor } \\
\text { agonists }\end{array}$ & 96,813 & 92 & 26 & $1.16(0.89,1.51)$ & $1.25(0.89,1.76)$ & $3(-5,13)$ \\
\hline Sulfonylureas & 142,578 & 157 & 23 & [reference] & [reference] & [reference] \\
\hline
\end{tabular}

${ }^{a}$ Adjusted for country, age, sex, place of birth and cohabitation status; history of cardiovascular disease, diabetes complications, cancer (more than 1 year before cohort entry), gallbladder or pancreatic disorders, liver disease, inflammatory bowel disease and alcohol-related disorders; as well as diabetes medications and measures of healthcare utilisation 
Fig. 2 Cumulative incidence of cholangiocarcinoma in the analysis of (a) DPP4 inhibitors and (b) GLP-1-receptor agonists, respectively, vs sulfonylureas. Because of declining numbers of patients at risk and outcome events, cumulative incidence curves were truncated at 10 years (maximum follow-up in the study was 12 years). GLP-1-RA, GLP1-receptor agonists
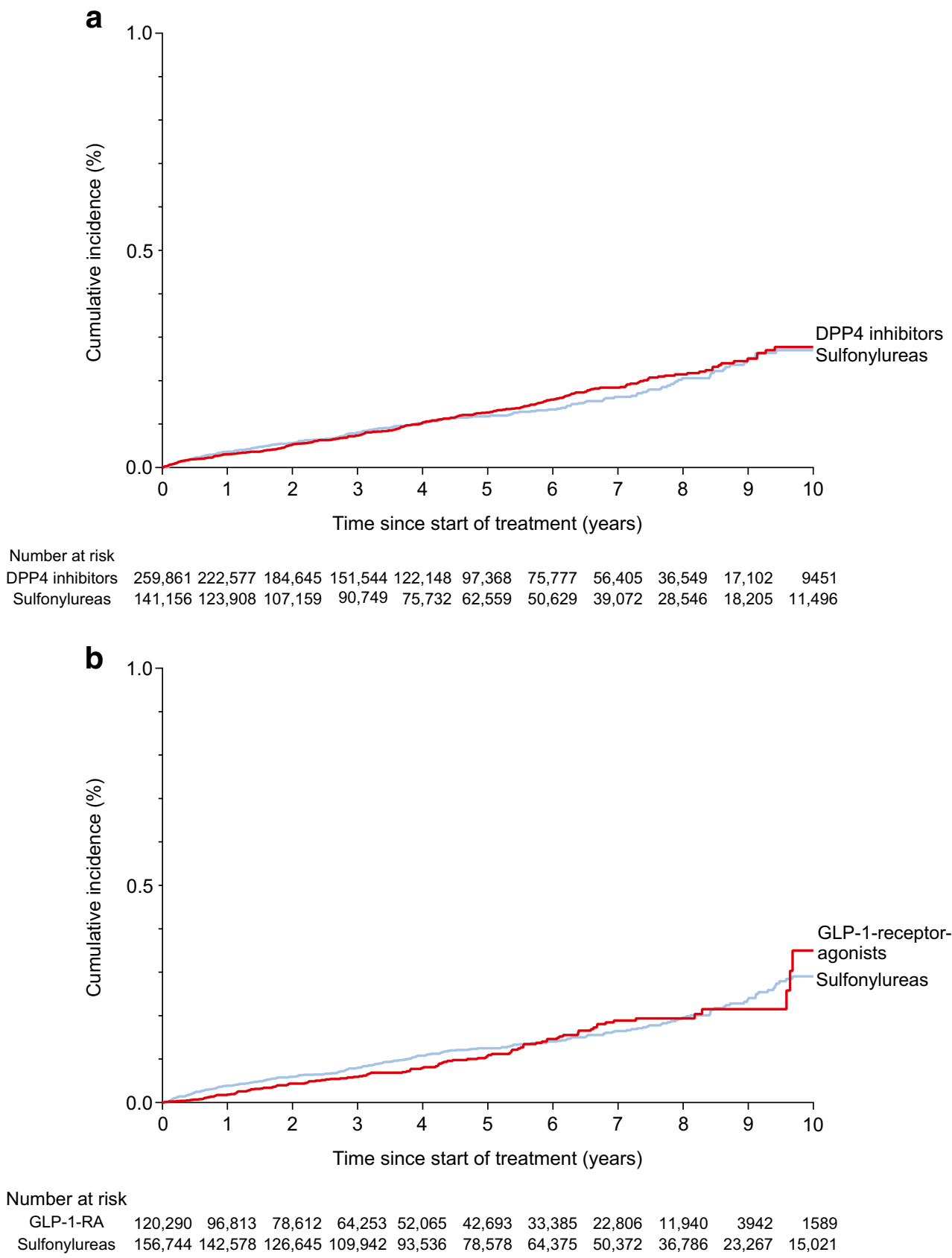

5.5 [3.2-8.1] years) remained at risk at 1 year after start of follow-up. During 1,036,587 person-years of follow-up, cholangiocarcinoma occurred among 92 patients with use of GLP1-receptor agonists (incidence rate, 26 per 100,000 patientyears) and among 157 patients with use of sulfonylureas (incidence rate, 23 per 100,000 patient-years). The adjusted HR for use of GLP-1-receptor agonists vs sulfonylureas was 1.25 $(95 \%$ CI $0.89,1.76)$ and the absolute difference was $3(95 \%$ CI -5, 13) events per 100,000 person-years. The adjusted HR was similar in all countries (ESM Table 4). The number and proportion of events by type of cholangiocarcinoma in the primary analyses are shown in ESM Table 5.
The additional analyses by time since treatment initiation are shown in ESM Table 6. None of the analyses showed statistically significant associations with cholangiocarcinoma. The point estimate increased for a time since treatment initiation of 3 to $<6$ years for DPP4 inhibitors but decreased again for $\geq 6$ years. In analyses of GLP-1-receptor agonists, the point estimate decreased with longer time since treatment initiation.

Sensitivity analyses Results from the sensitivity analyses are shown in Tables 3 and 4. Varying the lag period, the adjusted HR vs sulfonylureas was $1.09(95 \%$ CI $0.88,1.33)$ for DPP4 inhibitors and $1.11(95 \%$ CI $0.83,1.49)$ for GLP-1-receptor 
Table 3 Sensitivity analyses of the association between use of DPP4 inhibitors and risk of cholangiocarcinoma

\begin{tabular}{|c|c|c|c|c|c|c|}
\hline Analysis & Drug & $\mathrm{n}$ & Events & $\begin{array}{l}\text { Events per } 100,000 \\
\text { person-years }\end{array}$ & $\begin{array}{l}\text { Unadjusted HR } \\
(95 \% \mathrm{CI})\end{array}$ & $\begin{array}{l}\text { Adjusted }^{\mathrm{a}} \mathrm{HR} \\
(95 \% \mathrm{CI})\end{array}$ \\
\hline \multirow[t]{2}{*}{ No lag period } & DPP4 inhibitors & 259,861 & 296 & 27 & $1.04(0.86,1.26)$ & $1.09(0.88,1.33)$ \\
\hline & Sulfonylureas & 141,156 & 177 & 26 & [reference] & [reference] \\
\hline \multirow[t]{2}{*}{2 year lag period } & DPP4 inhibitors & 184,645 & 176 & 27 & $1.13(0.89,1.44)$ & $1.11(0.85,1.45)$ \\
\hline & Sulfonylureas & 107,159 & 105 & 24 & [reference] & [reference] \\
\hline \multirow{2}{*}{$\begin{array}{l}\text { Censor at use of GLP-1- } \\
\text { receptor agonists }\end{array}$} & DPP4 inhibitors & 209,240 & 184 & 26 & $1.18(0.93,1.49)$ & $1.16(0.89,1.50)$ \\
\hline & Sulfonylureas & 120,534 & 114 & 23 & [reference] & [reference] \\
\hline \multirow[t]{2}{*}{ Adjust for calendar year } & DPP4 inhibitors & 222,577 & 222 & 26 & $1.13(0.91,1.41)$ & $1.14(0.89,1.46)$ \\
\hline & Sulfonylureas & 123,908 & 128 & 23 & [reference] & [reference] \\
\hline \multirow{2}{*}{$\begin{array}{l}\text { First treatment episode with traditional } \\
\text { active-comparator new-user design }\end{array}$} & DPP4 inhibitors & 131,298 & 118 & 25 & $1.12(0.88,1.42)$ & $1.16(0.90,1.49)$ \\
\hline & Sulfonylureas & 133,233 & 172 & 24 & [reference] & [reference] \\
\hline \multirow[t]{2}{*}{ SGLT2 inhibitors as comparator } & DPP4 inhibitors & 127,842 & 66 & 24 & $1.05(0.59,1.86)$ & $0.95(0.50,1.80)$ \\
\hline & $\begin{array}{l}\text { SGLT2 } \\
\quad \text { inhibitors }\end{array}$ & 38,667 & 14 & 23 & [reference] & [reference] \\
\hline \multirow{2}{*}{$\begin{array}{l}\text { Not applying exclusion criteria } \\
\text { related to risk factors of } \\
\text { cholangiocarcinoma }\end{array}$} & DPP4 inhibitors & 231,414 & 237 & 27 & $1.15(0.93,1.43)$ & $1.15(0.91,1.45)$ \\
\hline & Sulfonylureas & 129,024 & 134 & 23 & [reference] & [reference] \\
\hline
\end{tabular}

${ }^{a}$ Adjusted for country, age, sex, place of birth and cohabitation status; history of cardiovascular disease, diabetes complications, cancer (more than 1 year before cohort entry), gallbladder or pancreatic disorders, liver disease, inflammatory bowel disease and alcohol-related disorders; as well as diabetes medications and measures of healthcare utilisation. Analyses using SGLT2 inhibitors as the comparator were also adjusted for use of sulfonylureas. In analyses in which exclusion criteria related to risk factors of cholangiocarcinoma were not applied, healthcare visit for any cancer (except non-melanoma skin cancer) within the previous year was included in the variable for history of cancer; HIV, hepatitis B or C, congenital cystic disease of the liver or choledochal duct, primary sclerosing cholangitis and cystic fibrosis before cohort entry were adjusted for as a single variable due to the low number of risk factor-exposed cases

agonists without a lag period and 1.11 (95\% CI $0.85,1.45)$ for DPP4 inhibitors and 1.06 (95\% CI 0.72, 1.57) for GLP-1receptor agonists with a 2 year lag period. When follow-up in the analysis of DPP4 inhibitors was censored at initiation of GLP-1-receptor agonists, the adjusted HR was 1.16 (95\% CI $0.89,1.50)$. The corresponding HR of GLP-1-receptor agonists when follow-up was censored at initiation of DPP4 inhibitors was 1.21 (95\% CI 0.82, 1.80). In analyses additionally adjusted for calendar year, the adjusted HR was 1.14 (95\% CI $0.89,1.46)$ for DPP4 inhibitors and 1.25 (95\% CI $0.88,1.78$ ) for GLP-1-receptor agonists. The analyses based on a traditional active-comparator new-user design using the first treatment episode during the study period included 131,298 new users of DPP4 inhibitors vs 133,233 new users of sulfonylureas and 53,302 new users of GLP-1-receptor agonists vs 146,294 new users of sulfonylureas who remained at risk at 1 year after start of follow-up (ESM Fig. 1; baseline characteristics in ESM Table 7). Hence, in these cohorts, the eligibility criterion requiring no use of the incretin-based drug of interest and the comparator prior to cohort entry led to a substantial exclusion of patients initiating an incretin-based drug because of a history of previous sulfonylureas use. In these analyses, the adjusted HR was 1.16 (95\% CI 0.90, 1.49) for DPP4 inhibitors and 1.39 (95\% CI 0.94, 2.07) for GLP-1-receptor agonists. The analyses using SGLT2 inhibitors as the comparator included 127,842 new users of DPP4 inhibitors (median [IQR] follow-up time, 3.5 [2.2-4.9] years) vs 38,667 new users of SGLT2 inhibitors (median [IQR] follow-up time, 2.5 [1.7-3.7] years) and 51,064 new users of GLP-1-receptor agonists (median [IQR] follow-up time, 3.2 [2.0-4.7] years) vs 57,736 new users of SGLT2 inhibitors (median [IQR] follow-up time, 2.5 [1.7-3.8] years) who remained at risk at 1 year after start of follow-up (ESM Fig. 2; baseline characteristics in ESM Table 8). In these analyses the adjusted HR was $0.95(95 \%$ CI $0.50,1.80)$ for DPP4 inhibitors and 0.96 (95\% CI 0.52, 1.78) for GLP-1-receptor agonists. In the analyses in which the exclusion criteria related to risk factors of cholangiocarcinoma were not applied, the adjusted HR vs sulfonylureas was 1.15 (95\% CI 0.91, 1.45) for DPP4 inhibitors and 1.19 (95\% CI 0.85, 1.66) for GLP-1receptor agonists.

\section{Discussion}

We used nationwide registers in three Scandinavian countries to conduct two cohort studies that assessed the risk of cholangiocarcinoma associated with use of DPP4 inhibitors and GLP-1receptor agonists, respectively. In analyses using sulfonylureas as the active comparator, there was no statistically significant increased risk of cholangiocarcinoma associated with either drug class over a median follow-up of 4.5 years. 
Table 4 Sensitivity analyses of the association between use of GLP-1-receptor agonists and risk of cholangiocarcinoma

\begin{tabular}{|c|c|c|c|c|c|c|}
\hline Analysis & Drug & $\mathrm{n}$ & Events & $\begin{array}{l}\text { Events per } \\
100,000 \text { person- } \\
\text { years }\end{array}$ & $\begin{array}{l}\text { Unadjusted HR } \\
(95 \% \mathrm{CI})\end{array}$ & $\begin{array}{l}\text { Adjusted }^{\mathrm{a}} \mathrm{HR} \\
(95 \% \mathrm{CI})\end{array}$ \\
\hline \multirow[t]{2}{*}{ No lag period } & GLP-1-receptor agonists & 120,290 & 111 & 24 & $0.93(0.74,1.17)$ & $1.11(0.83,1.49)$ \\
\hline & Sulfonylureas & 156,744 & 216 & 26 & [reference] & [reference] \\
\hline \multirow[t]{2}{*}{2 year lag period } & GLP-1-receptor agonists & 78,612 & 69 & 25 & $1.12(0.83,1.51)$ & $1.06(0.72,1.57)$ \\
\hline & Sulfonylureas & 126,645 & 130 & 24 & [reference] & [reference] \\
\hline \multirow[t]{2}{*}{ Censor at use of DPP4 inhibitors } & GLP-1-receptor agonists & 85,578 & 77 & 26 & $1.17(0.87,1.56)$ & $1.21(0.82,1.80)$ \\
\hline & Sulfonylureas & 123,882 & 118 & 23 & [reference] & [reference] \\
\hline \multirow[t]{2}{*}{ Adjust for calendar year } & GLP-1-receptor agonists & 96,813 & 92 & 26 & $1.16(0.89,1.51)$ & $1.25(0.88,1.78)$ \\
\hline & Sulfonylureas & 142,578 & 157 & 23 & [reference] & [reference] \\
\hline \multirow{2}{*}{$\begin{array}{l}\text { First treatment episode with traditional } \\
\text { active-comparator new-user design }\end{array}$} & GLP-1-receptor agonists & 53,302 & 50 & 27 & $1.21(0.88,1.66)$ & $1.39(0.94,2.07)$ \\
\hline & Sulfonylureas & 146,294 & 180 & 24 & [reference] & [reference] \\
\hline \multirow[t]{2}{*}{ SGLT2 inhibitors as comparator } & GLP-1-receptor agonists & 51,064 & 24 & 23 & $0.86(0.48,1.54)$ & $0.96(0.52,1.78)$ \\
\hline & SGLT2 inhibitors & 57,736 & 23 & 25 & [reference] & [reference] \\
\hline \multirow{2}{*}{$\begin{array}{l}\text { Not applying exclusion criteria } \\
\text { related to risk factors of } \\
\text { cholangiocarcinoma }\end{array}$} & GLP-1-receptor agonists & 99,449 & 94 & 26 & $1.14(0.88,1.47)$ & $1.19(0.85,1.66)$ \\
\hline & SGLT2 inhibitors & 148,454 & 165 & 24 & [reference] & [reference] \\
\hline
\end{tabular}

${ }^{\text {a }}$ Adjusted for country, age, sex, place of birth and cohabitation status; history of cardiovascular disease, diabetes complications, cancer (more than 1 year before cohort entry), gallbladder or pancreatic disorders, liver disease, inflammatory bowel disease and alcohol-related disorders; as well as diabetes medications and measures of healthcare utilisation. Analyses using SGLT2 inhibitors as the comparator were also adjusted for use of sulfonylureas. In analyses in which exclusion criteria related to risk factors of cholangiocarcinoma were not applied, healthcare visit for any cancer (except non-melanoma skin cancer) within the previous year was included in the variable for history of cancer; HIV, hepatitis B or C, congenital cystic disease of the liver or choledochal duct, primary sclerosing cholangitis and cystic fibrosis before cohort entry were adjusted for as a single variable due to the low number of risk factor-exposed cases

Data from clinical trials regarding the association between use of incretin-based drugs and cholangiocarcinoma are conflicting, with the limited number of events and follow-up time precluding conclusive analyses. In the Liraglutide Effect and Action in Diabetes: Evaluation of Cardiovascular Outcome Results (LEADER) trial of the GLP-1-receptor agonist liraglutide, more biliary cancers were observed among those receiving active treatment vs placebo (six vs two events), with all but one event in the liraglutide group being cholangiocarcinomas [11]. In the Saxagliptin Assessment of Vascular Outcomes Recorded in Patients with Diabetes Mellitus-Thrombolysis in Myocardial Infarction (SAVOR-TIMI) 53 trial, however, fewer cases of hepatobiliary cancers occurred among those receiving the DPP4 inhibitor saxagliptin vs placebo (nine vs 12); specific data for biliary cancers have not been reported [10]. A recent metaanalysis [9] identified three clinical trials of DPP4 inhibitors in which at least one case of cholangiocarcinoma was reported [12-14]; three and four events occurred among patients receiving DPP4 inhibitors and placebo, respectively.

An observational study by Abrahami et al. [8] has prompted the European Medicines Agency to monitor cholangiocarcinoma events with incretin-based drugs. The study found that use of DPP4 inhibitors, compared with other second- or third-line glucose-lowering drugs, was associated with a $77 \%$ increase in the risk of cholangiocarcinoma (HR 1.77 [95\% CI 1.04, 3.01]), although the analysis only included 27 DPP4 inhibitor-exposed events. Our analyses of DPP4 inhibitors included 222 exposed events and did not show any statistically significant association with cholangiocarcinoma (HR vs sulfonylureas 1.15 [95\% CI $0.90,1.46])$. In addition to the larger number of events in our study which allowed for analysis with more statistical power and higher precision, we used a different study population and study design; the analyses also differed in the covariates used for adjustment. Moreover, the findings of the two studies should be interpreted in the context of the uncertainty of the estimates. In the analyses of GLP-1-receptor agonists, the study by Abrahami et al. included only seven exposed events. Thus, the confidence intervals for the HR vs use of other second- or third-line glucoselowering drugs were wide, although the point estimate indicated that also these drugs might be associated with an increased risk (HR 1.97 [95\% CI 0.84, 4.66]). In our analyses of GLP-1receptor agonists, which included 92 exposed events, the HR vs use of sulfonylureas was 1.25 (95\% CI 0.89, 1.76). In our sensitivity analyses using SGLT2 inhibitors as the comparator, the HR was 0.95 (95\% CI 0.50, 1.80) for DPP4 inhibitors and $0.96(95 \%$ CI $0.52,1.78$ ) for GLP-1-receptor agonists. Nonetheless, both our analyses and those performed by Abrahami et al. indicate that potential absolute risk increases associated with use of incretinbased drugs, if any, are likely small. 
Strengths of our study include the inclusion of nationwide cohorts of patients seen in routine clinical practice in three countries and the use of national cancer registers in which the majority of cancer cases are morphologically verified [18-20]. Moreover, the median follow-up in the primary analyses was 4.5 years for DPP4 inhibitors with $25 \%$ of the patients (i.e. almost 65,000 patients) having more than 7.0 years of follow-up. The corresponding numbers for GLP-1-receptor agonists were 4.4 and 6.9 years. While there was no indication of an increased risk even after 6 years or more since treatment initiation in our additional analyses (HR for DPP4 inhibitors 0.92 [95\% CI 0.58, 1.47]; HR for GLP-1-receptor agonists 1.11 [95\% CI 0.54, 2.25]), incretinbased drugs can be used as lifelong treatments and studies with longer follow-up time would be needed when such data are available. Moreover, to mitigate the risk of confounding by indication, severity of disease and unmeasured participant characteristics, we used an active-comparator design assessing risk of cholangiocarcinoma with sulfonylureas in the primary analyses. An additional strength was the use of an alternative comparator, SGLT2 inhibitors, in sensitivity analyses; like the incretin-based drugs, these drugs were introduced in clinical practice more recently than sulfonylureas. Our study has limitations. The exposure definition was based on filled prescriptions. Low adherence may have biased the findings towards the null. Moreover, a study has suggested that biliary cancers might be underreported in the Swedish cancer register [23]. Potential underreporting is unlikely to differ between users of incretin-based drugs and sulfonylureas and, in our study, the incidence of cholangiocarcinoma was similar or higher in Sweden compared with in Denmark and Norway. Finally, due to the observational nature of this study, residual and unmeasured confounding cannot be ruled out.

In conclusion, in this study using nationwide data from three Scandinavian countries and sulfonylureas as the comparator, neither use of DPP4 inhibitors nor use of GLP-1-receptor agonists was associated with a significantly increased risk of cholangiocarcinoma.

Supplementary Information The online version contains peer-reviewed but unedited supplementary material available at https://doi.org/10.1007/ s00125-021-05508-1.

Data availability The datasets analysed during the current study are not publicly available because they are register-based and contain information about individual patients.

Funding Open access funding provided by Karolinska Institute. PU was supported by grants from the Swedish Heart-Lung Foundation and the Swedish Society for Medical Research. BP was supported by an investigator grant from the Strategic Research Area Epidemiology programme at Karolinska Institutet. The study was conducted with research grant support from the Swedish Cancer Society and the Nordic Cancer Union. The funders had no role in the design and conduct of the study; collection, management, analysis and interpretation of the data; or preparation, review or approval of the manuscript. The corresponding author had full access to all the data in the study and had final responsibility for the decision to submit for publication.
Authors' relationships and activities CJ reports personal fees from Pfizer and Bayer outside the submitted work. BE reports personal fees from Amgen, AstraZeneca, Boehringer Ingelheim, Eli Lilly, Merck Sharp \& Dohme, Mundipharma, Navamedic, Novo Nordisk and RLS Global outside the submitted work, and grants from Sanofi outside the submitted work. SG reports lecture fees and research grants from AstraZeneca, Boehringer Ingelheim, Eli Lilly, Merck Sharp \& Dohme, Novo Nordisk and Sanofi outside of the submitted work. HS reports consulting fees from Celgene and employment at IQVIA outside the submitted work. The other authors declare that there are no relationships or activities that might bias, or be perceived to bias, their work.

Contribution statement PU, VW, HS and BP were responsible for the concept and design. All authors contributed to the acquisition, analysis or interpretation of data. PU, VW and BP wrote the first draft of the manuscript. All authors contributed to the critical revision of the manuscript for important intellectual content. VW performed the statistical analysis. All authors approved the final manuscript. PU and BP obtained funding. PU, VW and BP are responsible for the integrity of the work as a whole and are guarantors of the work.

Open Access This article is licensed under a Creative Commons Attribution 4.0 International License, which permits use, sharing, adaptation, distribution and reproduction in any medium or format, as long as you give appropriate credit to the original author(s) and the source, provide a link to the Creative Commons licence, and indicate if changes were made. The images or other third party material in this article are included in the article's Creative Commons licence, unless indicated otherwise in a credit line to the material. If material is not included in the article's Creative Commons licence and your intended use is not permitted by statutory regulation or exceeds the permitted use, you will need to obtain permission directly from the copyright holder. To view a copy of this licence, visit http://creativecommons.org/licenses/by/4.0/.

\section{References}

1. Cosentino F, Grant PJ, Aboyans V et al (2020) 2019 ESC guidelines on diabetes, pre-diabetes, and cardiovascular diseases developed in collaboration with the EASD. Eur Heart J 41:255-323. https://doi.org/10.1093/eurheartj/ehz486

2. American Diabetes Association (ADA) (2020) 9. Pharmacologic approaches to glycemic treatment: standards of medical care in diabetes-2020. Diabetes Care 43(Suppl 1):S98-S110. https:// doi.org/10.2337/dc20-S009

3. Arnett DK, Blumenthal RS, Albert MA et al (2019) 2019 ACC/ AHA guideline on the primary prevention of cardiovascular disease: a report of the American College of Cardiology/ American Heart Association Task Force on Clinical Practice Guidelines. Circulation 140:e596-e646. https://doi.org/10.1161/ CIR.0000000000000678

4. Rangaswami J, Bhalla V, de Boer IH et al (2020) Cardiorenal protection with the newer antidiabetic agents in patients with diabetes and chronic kidney disease: a scientific statement from the American Heart Association. Circulation 142:1-22. https://doi. org/10.1161/CIR.0000000000000920

5. Marzioni M, Alpini G, Saccomanno S et al (2007) Glucagon-like peptide-1 and its receptor agonist exendin-4 modulate cholangiocyte adaptive response to cholestasis. Gastroenterology 133:244-255. https://doi.org/10.1053/j. gastro.2007.04.007 
6. Chen B-D, Zhao W-C, Dong J-D et al (2014) Expression of GLP$1 \mathrm{R}$ protein and its clinical role in intrahepatic cholangiocarcinoma tissues. Mol Biol Rep 41:4313-4320. https://doi.org/10.1007/ s11033-014-3302-7

7. Marzioni M, Alpini G, Saccomanno S et al (2009) Exendin-4, a glucagon-like peptide 1 receptor agonist, protects cholangiocytes from apoptosis. Gut 58:990-997. https://doi.org/10.1136/gut. 2008.150870

8. Abrahami D, Douros A, Yin H et al (2018) Incretin based drugs and risk of cholangiocarcinoma among patients with type 2 diabetes: population based cohort study. BMJ 363:k4880. https://doi.org/10. 1136/bmj.k4880

9. Dicembrini I, Nreu B, Montereggi C et al (2020) Risk of cancer in patients treated with dipeptidyl peptidase- 4 inhibitors: an extensive meta-analysis of randomized controlled trials. Acta Diabetol 57: 689-696. https://doi.org/10.1007/s00592-020-01479-8

10. Leiter LA, Teoh H, Mosenzon O et al (2016) Frequency of cancer events with saxagliptin in the SAVOR-TIMI 53 trial. Diabetes Obes Metab 18:186-190. https://doi.org/10.1111/dom.12582

11. Nauck MA, Jensen TJ, Rosenkilde C et al (2018) Neoplasms reported with liraglutide or placebo in people with type 2 diabetes: results from the LEADER randomized trial. Diabetes Care 41: 1663-1671. https://doi.org/10.2337/dc17-1825

12. Green JB, Bethel MA, Armstrong PW et al (2015) Effect of sitagliptin on cardiovascular outcomes in type 2 diabetes. N Engl J Med 373:232-242. https://doi.org/10.1056/NEJMoa1501352

13. Gantz I, Chen M, Suryawanshi S et al (2017) A randomized, placebo-controlled study of the cardiovascular safety of the onceweekly DPP-4 inhibitor omarigliptin in patients with type 2 diabetes mellitus. Cardiovasc Diabetol 16:112. https://doi.org/10.1186/ s12933-017-0593-8

14. Yki-Järvinen H, Rosenstock J, Durán-Garcia S et al (2013) Effects of adding linagliptin to basal insulin regimen for inadequately controlled type 2 diabetes: a $\geq 52$-week randomized, double-blind study. Diabetes Care 36:3875-3881. https://doi.org/10.2337/dc122718

15. European Medicines Agency (2019) Pharmacovigilance Risk Assessment Committee (PRAC): Minutes of meeting on 14-17 January 2019. Available from https://www.ema.europa.eu/en/ documents/minutes/minutes-prac-meeting-14-17-january-2019 en.pdf. Accessed 21 Feb 2020

16. European Medicines Agency (2019) Pharmacovigilance Risk Assessment Committee (PRAC): Minutes of the meeting on 1114 June 2019. Available from https://www.ema.europa.eu/en/ documents/minutes/minutes-prac-meeting-11-14-june-2019_en. pdf. Accessed 21 Feb 2020

17. Inzucchi SE, Bergenstal RM, Buse JB et al (2015) Management of Hyperglycemia in type 2 diabetes, 2015: a patient-centered approach: update to a position statement of the American Diabetes Association and the European Association for the Study of Diabetes. Diabetes Care 38:140-149. https://doi.org/10.2337/ dc14-2441

18. Barlow L, Westergren K, Holmberg L et al (2009) The completeness of the Swedish Cancer Register - a sample survey for year 1998. Acta Oncol (Madr) 48:27-33. https://doi.org/10.1080/ 02841860802247664

19. Gjerstorff ML (2011) The Danish cancer registry. Scand J Public Health 39:42-45. https://doi.org/10.1177/1403494810393562

20. Larsen IK, Småstuen M, Johannesen TB et al (2009) Data quality at the Cancer Registry of Norway: an overview of comparability, completeness, validity and timeliness. Eur J Cancer 45:12181231. https://doi.org/10.1016/j.ejca.2008.10.037

21. Pottegård A, Friis S, Stürmer T et al (2018) Considerations for pharmacoepidemiological studies of drug-cancer associations. Basic Clin Pharmacol Toxicol 122:451-459. https://doi.org/10. 1111/bcpt. 12946

22. Lund JL, Richardson DB, Stürmer T (2015) The active comparator, new user study design in pharmacoepidemiology: historical foundations and contemporary application. Curr Epidemiol Rep 2:221228. https://doi.org/10.1007/s40471-015-0053-5

23. Kilander C, Mattsson F, Ljung R et al (2014) Systematic underreporting of the population-based incidence of pancreatic and biliary tract cancers. Acta Oncol (Madr) 53:822-829. https:// doi.org/10.3109/0284186X.2013.857429

Publisher's note Springer Nature remains neutral with regard to jurisdictional claims in published maps and institutional affiliations. 